\title{
The Blooming phenomenon: a rarity, but a dilemma in hysteroscopic resection of myomas
}

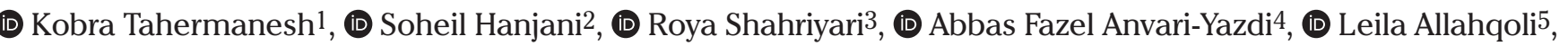 \\ (D) Ibrahim Alkatout 6 \\ 1Trauma and Injury Research Center, Iran University of Medical Sciences, Tehran, Iran \\ 2Department of Obstetrics and Gynecology, Good Samaritan Medical Center, Brockton, United States of America \\ 32Department of Obstetrics and Gynecology, Bank-e-Melli Iran Hospital, Tehran, Iran \\ 4Division of Biomedical Engineering, College of Engineering, University of Saskatchewan, Saskatoon, Saskatchewan, Canada \\ Iran University of Medical Sciences, Tehran, Iran \\ 6Department of Obstetrics and Gynecology, University Hospitals Schleswig-Holstein Campus Kiel, Kiel, Germany
}

\begin{abstract}
Modern surgical technologies allow gynecologists to treat most submucosal myomas hysteroscopically by some form of resection. What appears on imaging or direct visualization to be a submucosal myoma can be a single tumor, or may represent multiple smaller myomas appearing as one, compacted together in a typical pseudo capsule. During myoma resection, the effect of the media used to induce distension can vary, depending on the morphology of the myomas. After starting resection, the pressure of the distending media can push truly solitary myomas to somewhat flatten against the uterine wall. However, in the second type of myoma, the fluid can displace the myomas into the uterine cavity, an appearance similar to the blooming of a flower. The tip of the hysteroscope may enter the dissected spaces between the myomas, which impairs the panoramic view. This phenomenon may cause inadequate treatment of the myomas encountered during hysteroscopic myomectomy. In this study, the "Blooming phenomenon" is introduced, and the problems created by this phenomenon and solutions for its management are considered.
\end{abstract}

Keywords: Leiomyoma, submucosal myoma resection, fibroid, hysteroscopy

Received: 19 January 2021 Accepted: 22 March 2021

\section{Introduction}

Uterine leiomyomas, fibroids or myomas are the most common benign tumors in reproductive-age women in the world (1,2). Submucosal myomas (FIGO type 0, 1,2) that derive from myometrial cells just below the endometrium (3) are estimated to be the cause of $5-10 \%$ of cases of irregular bleeding, pain, subfertility and infertility $(4,5)$. The advancement in endoscopic surgical techniques has resulted in an improved ability to remove submucous uterine fibroids $(6,7)$. At present, the gold standard treatment for submucous myoma is hysteroscopic myomectomy (7). Different techniques and instruments have been introduced to facilitate the removal of submucosal myomas $(7,8)$. Since hysteroscopic morcellator devices, for example the Myosure, and/or other modern interventional and expensive facilities are not widely available, in many hospitals, submucosal myoma removal is still performed using a resectoscope (9). The removal of a submucosal myoma by resectoscope carries a greater risk than other techniques, because of the potential complications related to the procedure, such as cervix laceration, hemorrhage, uterine perforation, or clinical intravasation syndrome $(8,10,11)$. Studies have shown that the outcome of hysteroscopic submucosal myomectomy may be influenced by a number of factors, including the characteristics of the submucous myoma itself $(8,12)$, pseudocapsule fibroid, and by 
the techniques used to remove the myomas (8). The purpose of this article is to address the dilemma that we have termed "the Blooming phenomenon". The Blooming phenomenon may occur during hysteroscopic submucosal myomectomy with loop resection and can be associated with a number of clinical dilemmas and management problems during hysteroscopy that should be discussed.

\section{The Blooming phenomenon}

A pelvic sonography can show submucosal myomas in two different ways: a) genuinely solitary (Figure 1A); or b) apparently singular but in fact multiple myomas closely associated and compacted within a typical pseudo capsule (false solitary myoma) (Figure 1B). When submucosal myoma resection is performed for a genuinely solitary myoma, in some cases the pressure of the media used to induce uterine distension can lead to pressing and flattening of the myoma into and against the uterine wall (Figure 2). It may be necessary to reduce the pressure in order to allow the myoma to protrude more into the uterine cavity and become more visible. In the second type
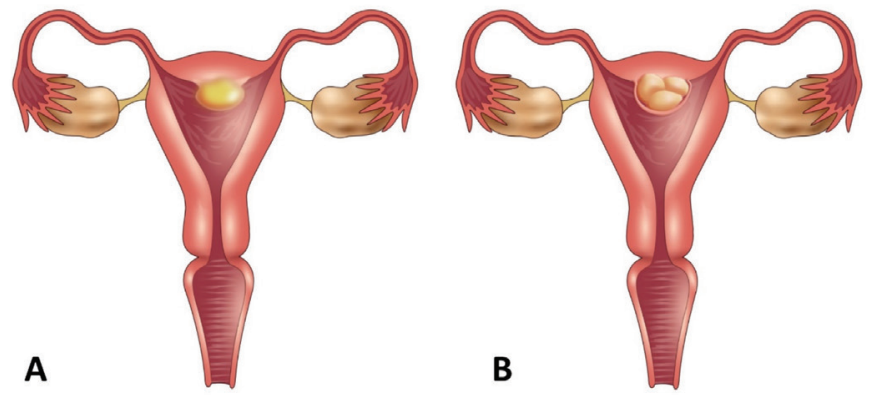

Figure 1. (A) Solitary submucosal myoma; and (B) apparently solitary, but actually multiple submucosal myomas
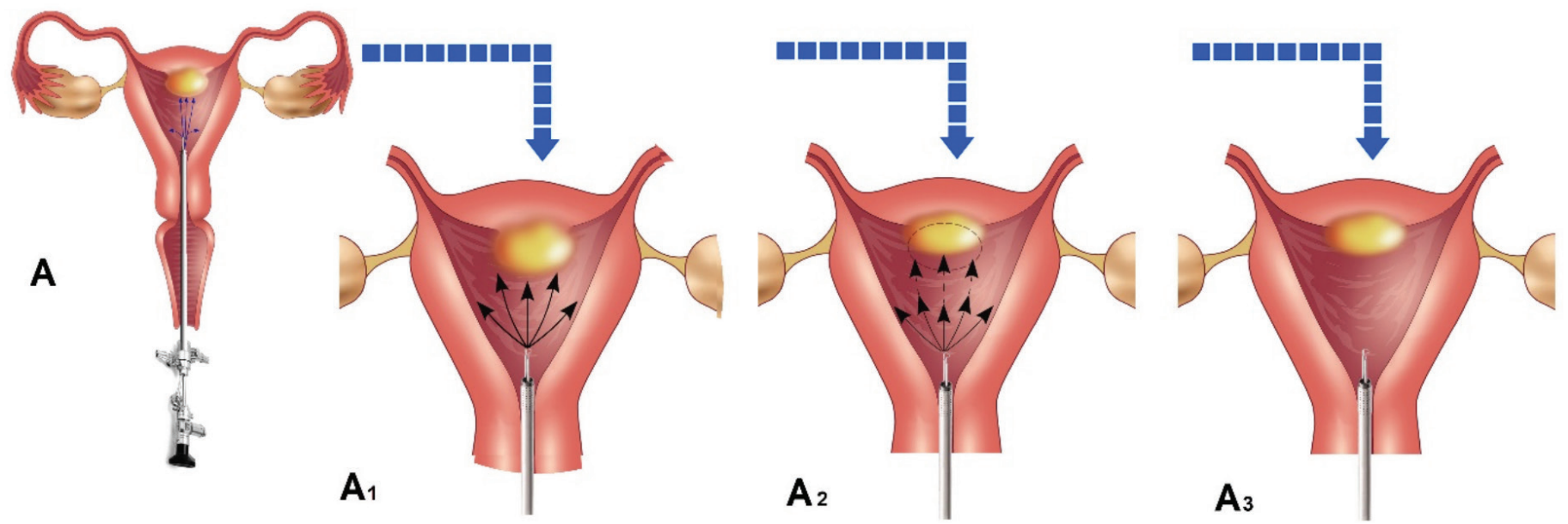

Figure 2. (A) Solitary submucosal myoma; $\left(A_{1}\right)$ arrows show the pressure of the distending media on the myoma; ( $A_{2}$ ) pressing and flattening of the true solitary myoma; and $\left(A_{3}\right)$ flattened solitary myoma due to pressure of distending media of myoma, once the resection begins and the pseudo capsule opens, the pressure of distension fluid entering the spaces between the myomas and the tendency of multiple myomas to disperse outwards when freed from the capsule, can result in the myomas extruding forward and laterally (Figure 3 ). This is similar to the blooming of a flower when the bud opens and the sepals, the small green leaf-like covering of the buds, are separated and the petals open. Therefore, the pressure of the distending media can produce different effects, depending on the type of submucous myoma (Figure 1).

Another consequence of the Blooming phenomenon is that it reduces the distance between the lens of the hysteroscope and the leading edge of the myomas, which can impair the panoramic view (Figure 4A). Furthermore, the tip of the hysteroscope may enter the dissected spaces between the myomas (Figure 4B). In this latter situation it may be necessary to stop operation before the myomas are completely removed or it may lead to inadvertent resection of the deeper areas of the myometrium and increase the risk of uterine wall perforation.

To manage the Blooming phenomenon, several steps are suggested:

i. The administration of 2-3 months of gonadotropin-releasing hormone agonist pre-operatively, when there is no specific pathology in the endometrium. This will usually decrease the size of myomas, leading to an improved panoramic view. A second consequence of this treatment is endometrial atrophy which can reduce the absorption of fluids during the procedure. ii. The use of ultrasound and/or magnetic resonance imaging may be helpful in differentiating genuinely solitary myomas from apparently singular myomas that are actually made up of a collection of smaller myomas.

iii. It is best to avoid small vertical or horizontal resections of 

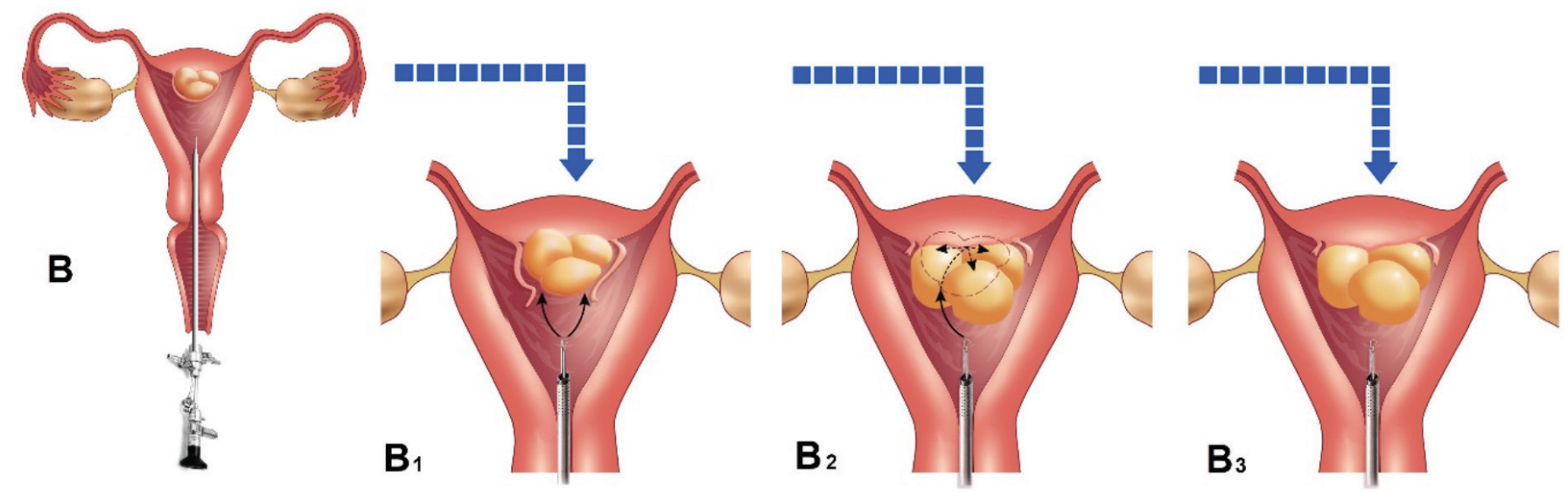

Figure 3. (B) resection of false solitary myoma; $\left(B_{1}\right)$ pseudo capsule opened due to resection; $\left(B_{2}\right)$ fluid entering the spaces between the myomas; and $\left(B_{3}\right)$ release of intracapsular compression leading to extrusion of the multiple small myomas by fluid displacement
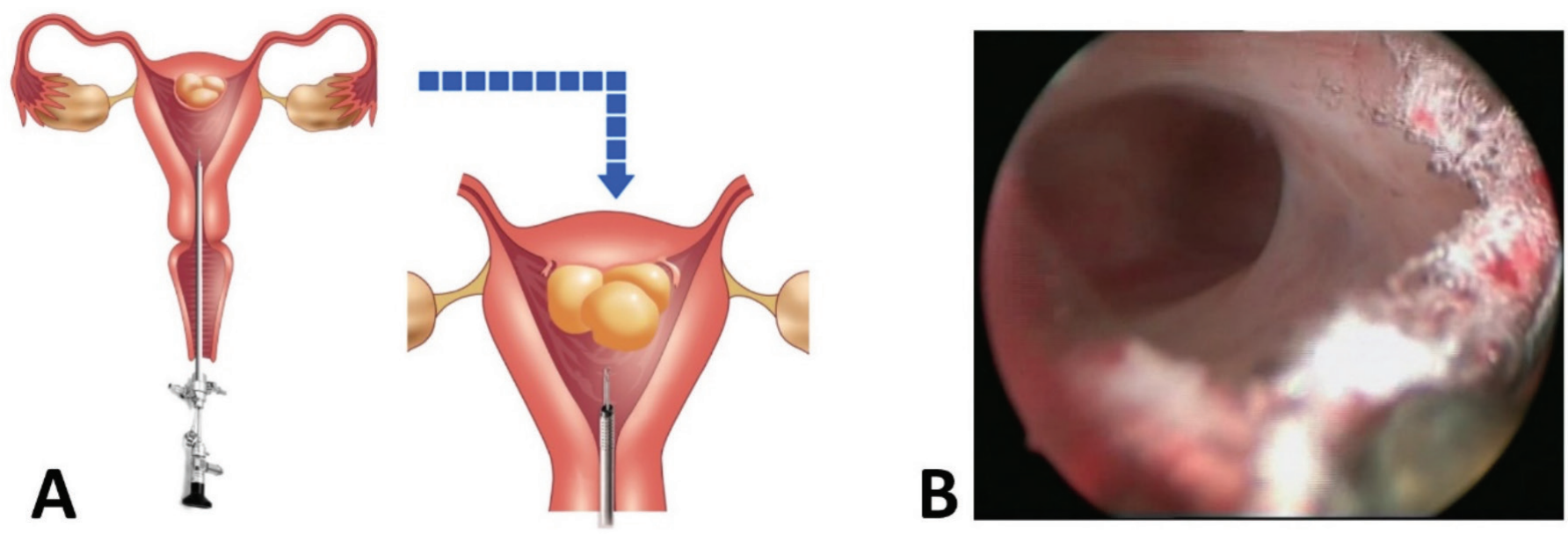

Figure 4. (A) schematic view of dissected spaces between the myomas; and (B) real hysteroscopic view of dissected myoma showing an inadvertent resection of the deeper areas of the myometrium

the myoma (Figure $5 \mathrm{~A}, \mathrm{~B}, \mathrm{C}, \mathrm{D}$ ), and it is better to place the tip of the resecting device near to the junction of myoma and the uterine wall and resect obliquely from the base to the tip (Figure 5E). This reduces the possibility that the myomas will protrude into the cavity and limit vision.

In summary, although the Blooming phenomenon is rare, when it does occur it can result in some clinical problems during a hysteroscopic resection. Therefore, surgeons should be aware of the existence of this phenomenon, to prevent potential complications and to know some techniques for the correct management, should they encounter the Blooming phenomenon. 

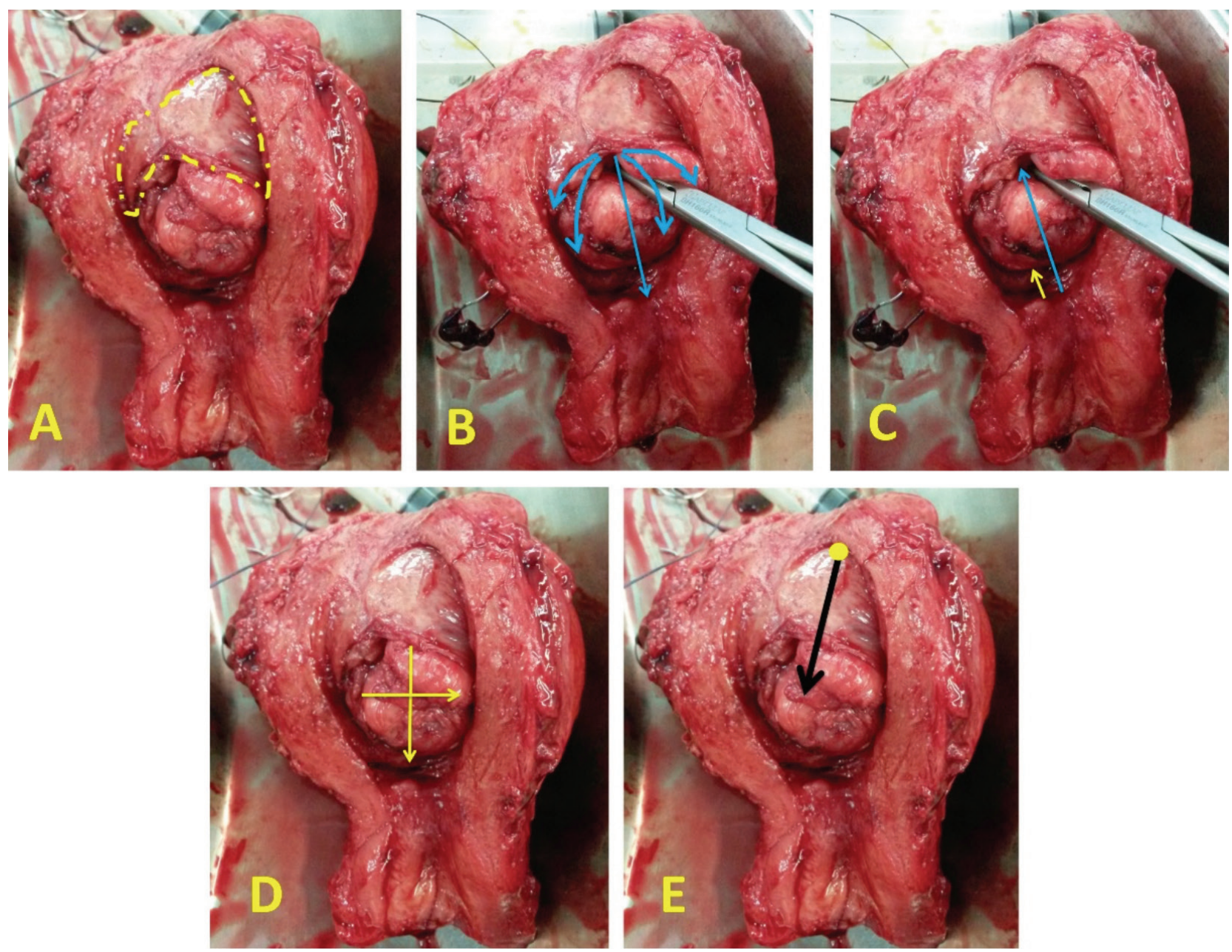

Figure 5. Hysterectomy of the same patient in Figure 4B with failed hysteroscopic myomectomy. (A) the yellow dotted line represents the pseudo capsule of the myoma; (B) blue arrows indicate the directions of protrusions of the myomas after partial resection of pseudo capsule; (C) blue arrow indicates the distance between tip of hysteroscope and myoma before dissection of pseudo capsule, while the yellow arrow demonstrates the reduction of this distance after dissection of pseudo capsule; (D) vertical and transverse resection direction of myoma which should be avoided; and (E) black arrow represents the correct direction of the resection

Acknowledgement: We thank Arman Nekounam for their valuable technical supports in graphical illustrations.

Video 1. The Blooming phenomenon during hysteroscopic resection of myoma

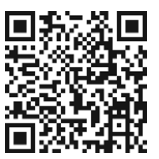

https://www.doi.org/10.4274/jtgga.galenos.2021.2021.0006.video1

Conflict of Interest: No conflict of interest was declared by the authors.
Financial Disclosure: The authors declared that this study received no financial support.

\section{References}

1. Casadio P, Guasina F, Morra C, Talamo M, Leggieri C, Frisoni J, et al. Hysteroscopic myomectomy: techniques and preoperative assessment. Minerva Ginecol 2016; 68: 154-66.

2. Alkatout I, Mettler L. Hysterectomy a comprehensive surgical approach. J Turk Ger Gynecol Assoc 2017; 18: 221-3.

3. Donnez J, Dolmans MM. Uterine fibroid management: from the present to the future. Hum Reprod Update 2016; 22: 665-86.

4. Sparic R, Mirkovic L, Malvasi A, Tinelli A. Epidemiology of uterine myomas: a review. Int J Fertil Steril 2016; 9: 424-35.

5. Alkatout I, Mettler L, Maass N, Noé GK, Elessawy M. Abdominal anatomy in the context of port placement and trocars. J Turk Ger Gynecol Assoc 2015; 16: 241-51. 
6. Saridogan E. Surgical treatment of fibroids in heavy menstrual bleeding. Womens Health 2016; 12: 53-62.

7. Osorio W, Posada N, Cano J, Tamayo S, Giraldo J. Hysteroscopic myomectomy for submucosal type 2 fibroids with cold enucleation technique and complete fibroid extraction using a double-lumen intracervical cannula. Fertil Steril 2021; 115: 522-4.

8. Tinelli A, Sparić R. Myoma pseudocapsule-a biological and surgical structure to respect during myomectomy. Srpski Arhiv Za Celokupno Lekarstvo 2020:148: 236-41.

9. Vitale SG, Sapia F, Rapisarda AMC, Valenti G, Santangelo F, Rossetti D, et al. Hysteroscopic morcellation of submucous myomas: a systematic review. BioMed Res Int 2017; 2017: 6848250.
10. Piecak K, Milart P. Hysteroscopic myomectomy. Prz Menopauzalny 2017; 16: 126-8.

11. Capmas P, Levaillant JM, Fernandez H. Surgical techniques and outcome in the management of submucous fibroids. Curr Opin Obstet Gynecol 2013; 25: 332-8.

12. Lasmar RB, Barrozo PR, Dias R, de Oliveira MA. Submucous myomas: a new presurgical classification to evaluate the viability of hysteroscopic surgical treatment--preliminary report. J Minim Invasive Gynecol 2005; 12: 308-11. 